\title{
Robust Control of Uncertain Nonlinear Systems
}

\author{
Guang-Hong Yang*, James Lam ${ }^{+}$and Jianliang Wang* \\ * School of EEE, Nanyang Technological University, Singapore 639798 \\ egyang@ntuvax.ntu.ac.sg, ejlwang@ntuvax.ntu.ac.sg \\ + Department of ME, The University of Hong Kong, Pokfulam Road, Hong Kong \\ jlam@hkuxa.hku.hk
}

\begin{abstract}
This paper considers robust $H_{\infty}$ control problems via state feedback and measurement feedback for a class of affine nonlinear systems with gain bounded uncertainties, and the solutions of the problems are derived in terms of smooth solutions of Hamilton-Jacobi inequalities. It is also shown that the results are extensions of the corresponding results for linear systems.
\end{abstract}

\section{Introduction}

In recent years, the $H_{\infty}$ control problem for nonlinear systems has been extensively investigated by several authors [1,3-5,10,11]. In particular, in [11] it was shown that the solution of the $H_{\infty}$ control problem via state feedback can be determined from the solution of a Hamilton-Jacobi equation (or inequality), which is the nonlinear version of the Riccati equation considered in [2] for the corresponding $H_{\infty}$ control problem for linear systems. Sufficient conditions for the solution of the problem in the case of measurement feedback have been given in a series of papers [1,3,4], and [1] and [5] discuss the necessity of these sufficient conditions.

This paper consider the robust $H_{\infty}$ control problem affine nonlinear systems with gain bounded uncertainty. Specifically, we consider an uncertain nonlinear system modeled by equations of the form

$$
\begin{aligned}
\dot{x} & =f(x)+\Delta f(x)+g_{1}(x) w+\left(g_{2}(x)+\Delta g_{2}(x)\right) u \\
z & =h_{1}(x)+k_{12}(x) u \\
y & =h_{2}(x)+\Delta h_{2}(x)+k_{21}(x) w
\end{aligned}
$$

where $x$ is a state vector defined on a neighbourhood $X$ of the origin in $R^{n}, u \in R^{m}$ denotes the control input, $w \in R^{r}$ the disturbance input, $z \in R^{s}$ the penalty variable and $y \in R^{p}$ the measured output. Functions $f(x), g_{1}(x), g_{2}(x), h_{1}(x), h_{2}(x), k_{12}(x)$ and $k_{21}(x)$ are known smooth (i.e. $C^{\infty}$ ) mappings defined in a neighbourhood of the origin in $R^{n}$ with $f(0)=0, h_{1}(0)=0$ and $h_{2}(0)=0$. The smooth uncertain mappings satisfy

0-7803-3590-2/96\$5.00 $\odot 1996$ IEEE $\triangle f(x) \in \Omega_{f}, \Delta g_{2}(x) \in \Omega_{g}$ and $\Delta h_{2}(x) \in \Omega_{h}$ with

$\Omega_{f} \triangleq\left\{f_{11}(x) \delta_{f}(x): \delta_{f}^{T}(x) \delta_{f}(x) \leq f_{22}^{T}(x) f_{22}(x), \forall x \in X\right\}$

$\Omega_{g} \triangleq\left\{g_{11}(x) \delta_{g}(x): \delta_{g}^{T}(x) \delta_{g}(x) \leq g_{22}^{T}(x) g_{22}(x), \forall x \in X\right\}$

$\Omega_{h} \triangleq\left\{h_{11}(x) \delta_{h}(x): \delta_{h}^{T}(x) \delta_{h}(x) \leq h_{22}^{T}(x) h_{22}(x), \forall x \in X\right\}$

where $f_{11}(x), g_{11}(x)$ and $h_{11}(x)$ are known smooth mappings, the smooth mappings $f_{22}(x), g_{22}(x)$ and $h_{22}(x)$ are given weighting mappings with $f_{22}(0)=0$ and $h_{22}(0)=0$.

Definition 1.1 ([9]) : Consider an uncertain nonlinear system $\Sigma$ given by

$$
\begin{aligned}
\dot{x} & =f(x)+\Delta f(x)+g(x) u \\
y & =h(x)
\end{aligned}
$$

with $x \in R^{n}, u \in R^{m}, y \in R^{p}$, and $\triangle f(x) \in \Omega_{f}$. Let $\gamma>0$ be given. The system $\Sigma$ is said to have locally robust disturbance attenuation performance $\gamma$ in $U \subset X\left(U\right.$ is a neighbourhood of the origin in $\left.R^{n}\right)$ if for any $\triangle f(x) \in \Omega_{f}$, the free system with $u=0$ is locally asymptotically stable with domain of attraction containing $U$, and the system $\Sigma$ is with an $L_{2}$ gain less than or equal to $\gamma$, i.e.,

$$
\int_{0}^{T}\|y(t)\|^{2} d t \leq \gamma^{2} \int_{0}^{T}\|u(t)\|^{2} d t
$$

for every $T>0$ and $u \in D$ with $D$ being defined as

$D=\left\{u: u \in L_{2}(0, T) \mid\right.$ if $x(0)=0, x(t) \in U$ for $\left.t \leq T\right\}$

where $L_{2}(0, T)$ denotes the set of vector-valued function $u(t)$ satisfying $\int_{0}^{T}\|u(t)\|^{2} d t<\infty$.

In this paper, the following two problems for the system (1) will be addressed.

Robust disturbance attenuation problem via state feedback: Given the system (1), find a state 
feedback controller

$$
u=\alpha(x), \quad \alpha(0)=0
$$

such that the resulting closed-loop system has local robust disturbance attenuation performance $\gamma$.

Robust disturbance attenuation problem via measurement feedback: Given the system (1), find a controller with the following form

$$
\begin{aligned}
\dot{\xi} & =\eta(\xi, y) \\
u & =\theta(x)
\end{aligned}
$$

such that the resulting closed-loop system has local robust disturbance attenuation performance $\gamma$, where $\xi$ is defined on a neighbourhood $V$ of the origin in $R^{v}$, and $\eta: V \times R^{p} \rightarrow R^{v}$, and $\theta: \rightarrow R^{m}$ are smooth functions with $\eta(0,0)=0$ and $\theta(0)=0$.

It should be noted that nonlinear models of the form (1) can be used to represent many real physical systems $[6,7,12]$. The uncertainty structure in equations (2)-(4) has been used in [9], and it was shown that the solution of the problem of robust disturbance attenuation via state feedback for the system of the form (1) with $\triangle g_{2}(x)=0$ and $\Delta h_{2}(x)=0$ is related to the existence of solution of a Hamilton-Jacobi inequality. The purpose of the present paper is to extend the result in [9] to the more general class of systems given by (1).

The following assumptions will be used in the sequel.

Assumption A1: The matrix $g_{22}^{T}(x) g_{22}(x)+$ $k_{12}^{T}(x) k_{12}(x)$ is nonsingular for each $x \in X$.

Assumption A2: For each $\Delta f(x) \in \Omega_{f}, \Delta g_{2}(x) \epsilon$ $\Omega_{g}$, any bounded trajectory $x(t)$ of the system

$\dot{x}(t)=f(x(t))+\Delta f(x(t))+\left[g_{2}(x(t))+\Delta g_{2}(x(t))\right] u(t)$

satisfying

$$
h_{1}(x(t))+k_{12}(x(t)) u(t)=0
$$

for all $t \geq 0$, is such that $\lim _{t \rightarrow \infty} x(t)=0$.

Assumption A3: The matrix $h_{11}(x) h_{11}^{T}(x)+$ $k_{21}(x) k_{21}^{T}(x)$ is nonsingular for each $x \in X$.

\section{Robust disturbance attenuation via state feedback}

The following results present sufficient conditions for the solvability of the problem of robust disturbance attenuation via state feedback for the system (1).

Theorem 2.1 Consider the system (1) and suppose Assumptions A1 and A2 hold. Suppose there exist scalar smooth functions $\lambda_{1}(x)>0$ and $\lambda_{2}(x)>0$ such that the Hamilton-Jacobi inequality

$$
\begin{aligned}
& H_{s}\left(x, V_{x}^{T}, \lambda_{1}(x), \lambda_{2}(x)\right) \\
& \triangleq V_{x} \tilde{f}(x)+\widetilde{h}_{1}^{T}(x) \widetilde{h}_{1}(x)+\frac{1}{4} V_{x} \widetilde{R}(x) V_{x}^{T} \leq 0
\end{aligned}
$$

has a $C^{1}$ positive definite solution $V(x)$, which is defined locally defined in a neighbourhood of $x=0$ and vanishing at $x=0$, where $V_{x}=\frac{\partial V}{\partial x}$ is the Jacobian matrix of $V$, and

$$
\begin{array}{r}
\tilde{f}(x)=f(x)-g_{2}(x) R_{2}^{-1}(x) k_{12}^{T}(x) h_{1}(x) \\
\widetilde{h}_{1}(x)=\left[\begin{array}{c}
\frac{1}{\sqrt{\lambda_{1}(x)}} f_{22}(x) \\
\frac{1}{\sqrt{\lambda_{2}(x)}} g_{22}(x) R_{2}^{-1}(x) k_{12}^{T}(x) h_{1}(x) \\
h_{1}(x)-k_{12}(x) R_{2}^{-1}(x) k_{12}^{T}(x) h_{1}(x)
\end{array}\right] \\
\widetilde{R}(x)=\frac{1}{\gamma^{2}} g_{1}(x) g_{1}^{T}(x)+\lambda_{1}(x) f_{11}(x) f_{11}^{T}(x) \\
+\lambda_{2}(x) g_{11}(x) g_{11}^{T}(x)-g_{2}(x) R_{2}^{-1}(x) g_{2}^{T}(x)
\end{array}
$$

with

$$
R_{2}(x)=\frac{1}{\lambda_{2}(x)} g_{22}^{T}(x) g_{22}(x)+k_{12}^{T}(x) k_{12}(x)
$$

Then, a solution of the robust disturbance attenuation problem via state feedback is given by

$$
u(x)=\alpha(x)=-R_{2}^{-1}(x)\left(\frac{1}{2} g_{2}^{T}(x) V_{x}^{T}+k_{12}^{T}(x) h_{1}(x)\right)
$$

Theorem 2.2 Consider the system (1) and suppose hypothesis A1 holds. Suppose there exist scalar smooth functions $\lambda_{1}(x)>0$ and $\lambda_{2}(x)>0$ such that the strict Hamilton-Jacobi inequality

$$
H_{s}\left(x, V_{x}^{T}, \lambda_{1}(x), \lambda_{2}(x)\right)<0 \text { for } x \neq 0
$$

has a $C^{1}$ positive definite solution $V(x)$, which is defined locally defined in a neighbourhood of $x=0$ and vanishing at $x=0$. Then, a solution of the robust disturbance attenuation problem via state feedback is given by (13).

Proof of Theorem 2.1. For any $\triangle f(x) \in \Omega_{f}, \Delta g_{2}(x) \epsilon$ $\Omega_{g}, \lambda_{1}(x)>0$, and $\lambda_{2}(x)>0$, from (2) and (3), we have

$$
\begin{gathered}
V_{x}\left(f(x)+\Delta f(x)+g_{1}(x) w+\left(g_{2}(x)+\Delta g_{2}(x)\right) u\right) \\
+\left\|h_{1}(x)+k_{12}(x) u\right\|^{2}-\gamma^{2}\|w\|^{2} \\
=V_{x} f(x)+V_{x} g_{1}(x) w+V_{x} g_{2}(x) u+\left\|h_{1}(x)+k_{12}(x) u\right\|^{2} \\
-\gamma^{2}\|w\|^{2}+V_{x} f_{11}(x) \delta_{f}(x)+V_{x} g_{11}(x) \delta_{g}(x) u \\
\leq V_{x} f(x)+V_{x} g_{1}(x) w+V_{x} g_{2}(x) u+\left\|h_{1}(x)+k_{12}(x) u\right\|^{2}
\end{gathered}
$$




$$
\begin{gathered}
-\gamma^{2}\|w\|^{2}+\frac{1}{4} V_{x}\left(\lambda_{1}(x) f_{11}(x) f_{11}^{T}(x)+\lambda_{2}(x) g_{11}(x) g_{11}^{T}(x)\right) V_{x}^{T} \\
+\frac{1}{\lambda_{1}(x)} f_{22}^{T}(x) f_{22}(x)+\frac{1}{\lambda_{2}(x)} u^{T} g_{22}^{T}(x) g_{22}(x) u \\
V_{x} f(x)+\frac{1}{4} V_{x}\left(\frac{1}{\gamma^{2}} g_{1}(x) g_{1}^{T}(x)+\lambda_{1}(x) f_{11}(x) f_{11}^{T}(x)\right. \\
\left.+\lambda_{2}(x) g_{11}(x) g_{11}^{T}(x)\right) V_{x}^{T}-\gamma^{2}\left\|w-\frac{1}{2 \gamma^{2}} g_{1}^{T}(x) V_{x}^{T}\right\|^{2} \\
+\frac{1}{\lambda_{1}(x)} f_{22}^{T}(x) f_{22}(x)+h_{1}^{T}(x) h_{1}(x) \\
+u^{T}\left(\frac{1}{\lambda_{2}(x)} g_{22}^{T}(x) g_{22}(x)+k_{12}^{T}(x) k_{12}(x)\right) u \\
+V_{x} g_{2}(x) u+2 u^{T} k_{12}^{T}(x) h_{1}(x)
\end{gathered}
$$

By Assumption A1, we know that the matrix $R_{2}(x)$ is positive definite for $\lambda_{2}(x)>0$, which further implies from (8)-(13) that

$$
\begin{gathered}
V_{x}\left[f(x)+\Delta f(x)+g_{1}(x) w+\left(g_{2}(x)+\Delta g_{2}(x)\right) u\right] \\
+\left\|h_{1}(x)+k_{12}(x) u\right\|^{2}-\gamma^{2}\|w\|^{2} \\
\leq V_{x} f(x)+h_{1}^{T}(x) h_{1}(x)+\frac{1}{\lambda_{1}(x)} f_{22}^{T}(x) f_{22}(x) \\
+\frac{1}{4} V_{x}\left(\frac{1}{\gamma^{2}} g_{1}(x) g_{1}^{T}(x)+\lambda_{1}(x) f_{11}(x) f_{11}^{T}(x)\right. \\
\left.+\lambda_{2}(x) g_{11}(x) g_{11}^{T}(x)\right) V_{x}^{T}-\gamma^{2}\left\|w-\frac{1}{2 \gamma^{2}} g_{1}^{T}(x) V_{x}^{T}\right\|^{2} \\
-\alpha^{T}(x) R_{2}(x) \alpha(x)+(u-\alpha(x))^{T} R_{2}(x)(u-\alpha(x)) \\
=V_{x}\left(f(x)-g_{2}(x) R_{2}^{-1}(x) k_{12}^{T}(x) h_{1}(x)\right)+h_{1}^{T}(x) h_{1}(x) \\
+\frac{1}{\lambda_{1}(x)} f_{22}^{T}(x) f_{22}(x)-h_{1}^{T}(x) k_{12}(x) R_{2}^{-1}(x) k_{12}(x) h_{1}(x) \\
+\frac{1}{4} V_{x}\left(\frac{1}{\gamma^{2}} g_{1}(x) g_{1}^{T}(x)+\lambda_{1}(x) f_{11}(x) f_{11}^{T}(x)\right. \\
\left.+\lambda_{2}(x) g_{11}(x) g_{11}^{T}(x)-g_{2}^{T}(x) R_{2}^{-1}(x) g_{2}(x)\right) V_{x}^{T} \\
+(u-\alpha(x))^{T} R_{2}(x)(u-\alpha(x))-\gamma^{2}\left\|w-\frac{1}{2 \gamma^{2}} g_{1}^{T}(x) V_{x}^{T}\right\|^{2} \\
=H_{s}\left(x, V_{x}^{T}, \lambda_{1}(x), \lambda_{2}(x)\right)+(u-\alpha(x))^{T} R_{2}(x)(u-\alpha(x)) \\
\quad-\gamma^{2}\left\|w-\frac{1}{2 \gamma^{2}} g_{1}^{T}(x) V_{x}^{T}\right\|^{2}
\end{gathered}
$$

By inequality (8) and $u=\alpha(x)$, we have

$$
\begin{aligned}
V_{x}(f(x)+\Delta f(x)+ & \left.\left(g_{2}(x)+\Delta g_{2}(x)\right) \alpha(x)\right)+V_{x} g_{1}(x) w \\
& \leq \gamma^{2}\|w\|^{2}-\|z\|^{2}
\end{aligned}
$$

which implies from Assumption A2 and the results in [4] that the closed-loop system

$$
\begin{aligned}
& \dot{x}=f(x)+\Delta f(x)+\left(g_{2}(x)+\Delta g_{2}(x)\right) \alpha(x)+g_{1}(x) w \\
& z=h_{1}(x)+k_{12}(x) \alpha(x)
\end{aligned}
$$

is with an $L_{2}$ gain which is less than or equal to $\gamma$, and asymptotically stable for any $\Delta f(x) \in \Omega_{f}$ and $\triangle g_{2}(x) \in \Omega_{g}$.

Proof of Theorem 2.2. By inequalities (14) and (15), we have

$$
\begin{gathered}
V_{x}\left(f(x)+\Delta f(x)+\left(g_{2}(x)+\Delta g_{2}(x)\right) \alpha(x)\right)+V_{x} g_{1}(x) w \\
<\gamma^{2}\|w\|^{2}-\|z\|^{2} \text { for } x \neq 0
\end{gathered}
$$

Thus, the conclusion of Theorem 2.2 is immediate from the results in [4].

Remark 2.1 When $\Delta g_{2}(x)=0$, Theorem 2.1 coincides with Theorem 2 in [9].

By Propositions 2.1 and 2.2 in [4], it is easy to see that the state feedback control law given by Theorem 2.1 or Theorem 2.2 also is a solution for the problem of local disturbance attenuation with internal stability for the following extended system $\Sigma_{s}$ :

$$
\begin{aligned}
& \dot{x}=f(x)+g_{2}(x) u \\
& +\left[\begin{array}{lll}
\gamma \sqrt{\lambda_{1}(x)} f_{11}(x) & \gamma \sqrt{\lambda_{2}(x)} g_{11}(x) & g_{1}(x)
\end{array}\right] \bar{w} \\
& \bar{z}=\left[\begin{array}{c}
\frac{1}{\sqrt{\lambda_{1}(x)}} f_{22}(x) \\
0 \\
h_{1}(x)
\end{array}\right]+\left[\begin{array}{c}
0 \\
\frac{1}{\sqrt{\lambda_{2}(x)}} g_{22}(x) \\
k_{12}(x)
\end{array}\right]
\end{aligned}
$$

Next section will discuss the relation between the uncertain system (1) and the extended system $\Sigma_{s}$ without uncertainty. In particular, the controller given by Theorem 2.2 guarantees that the resulting closed-loop system is uniformly asymptotically stable, which corresponds to the quadratic stability for an uncertain linear system if $V(x)=x^{T} P x$ for some positive definite matrix $P$.

In linear case, consider the uncertain linear system

$$
\begin{aligned}
\dot{x} & =\left(A+D_{1} F_{1}(t) E_{1}\right) x+B_{1} w+\left(B_{2}+D_{2} F_{2}(t) E_{2}\right) u \\
z & =C_{1} x+D_{12} u
\end{aligned}
$$

where the uncertainties $F_{1}(t)$ and $F_{2}(t)$ satisfy $F_{1}^{T}(t) F_{1}(t) \leq I$ and $F_{2}^{T}(t) F_{2}(t) \leq I$.

Then, for the quadratic stabilizability with an $H_{\infty}$ norm bound $\gamma([13])$ of the uncertain system (17), we have the following corollary.

Corollary 2.1 Suppose that the matrix $E_{2}^{T} E_{2}+$ $D_{12}^{T} D_{12}$ is positive definite. Then the uncertain system (17) is quadratically stabilizable with an $H_{\infty}$-norm bound $\gamma>0$ if there exist constants $\epsilon_{1}>0$ and $\epsilon_{2}>0$ such that the following Riccati inequality

$$
H_{l s}\left(Q, \epsilon_{1}, \epsilon_{2}\right)=\bar{A}^{T} Q+Q \bar{A}+\bar{C}_{1}^{T} \bar{C}_{1}+Q \bar{R} Q<0
$$


has a positive definite solution $Q$, where

$$
\begin{aligned}
& \bar{A}=A-B_{2} R_{2}^{-1}\left(\epsilon_{2}\right) D_{12}^{T} C_{1} \\
& \frac{1}{\sqrt{\epsilon_{1}}} E_{1} \\
& \bar{C}_{1}=\left[\begin{array}{c}
\frac{1}{\sqrt{\epsilon_{2}}} E_{2} R_{2}^{-1}\left(\epsilon_{2}\right) D_{12}^{T} C_{1} \\
C_{1}-D_{12} R_{2}^{-1}\left(\epsilon_{2}\right) D_{12}^{T} C_{1}
\end{array}\right] \\
& \bar{R}=\frac{1}{\gamma^{2}} B_{1} B_{1}^{T}+\epsilon_{1} D_{1} D_{1}^{T}+\epsilon_{2} D_{2} D_{2}^{T}-B_{2} R_{2}^{-1}\left(\epsilon_{2}\right) B_{2}^{T}
\end{aligned}
$$

with

$$
R_{2}\left(\epsilon_{2}\right)=\frac{1}{\epsilon_{2}} E_{2}^{T} E_{2}+D_{12}^{T} D_{12}
$$

Moreover, a suitable feedback control law is given by

$$
u=-R_{2}^{-1}\left(\epsilon_{2}\right)\left(B_{2}^{T} Q+D_{12} C_{1}\right)
$$

Proof. It is immediate from Theorem 2.2.

Remark 2.2 It should be noted that the Riccati inequality (18) involves the choices of two parameters $\epsilon_{1}$ and $\epsilon_{2}$. When choosing $\epsilon=\epsilon_{1}=\epsilon_{2}$, from Theorem 3.1 in [13], the condition under which the Riccati inequality

$$
H_{l s}(Q, \epsilon, \epsilon)<0
$$

has a positive definite solution $Q$ is sufficient and necessary for the quadratic stabilizability with an $H_{\infty}$-norm bound $\gamma$ of the following system

$$
\begin{aligned}
\dot{x} & =(A+\triangle A(t)) x+B_{1} w+\left(B_{2}+\triangle B_{2}(t)\right) u \\
z & =C_{1} x+D_{12} u
\end{aligned}
$$

where

$$
\left[\Delta A(t) \quad \Delta B_{2}(t)\right]=\left[\begin{array}{ll}
D_{1} & D_{2}
\end{array}\right] F(t)\left[\begin{array}{cc}
E_{1} & 0 \\
0 & E_{2}
\end{array}\right]
$$

with $F^{T}(t) F(t) \leq I$. By the notion of overbounding in [8], the system (20) is an overbounding system of the system (17), so the condition given by (19) only is a sufficient condition for the quadratic stabilizability with an $H_{\infty}$-norm bound $\gamma$ of the system (17), and applying it to the system (17) may bring about some conservativeness. The condition given by (18) may be less conservative, but the necessity of the condition is a problem to be investigated.

\section{Robust disturbance attenuation via measurement feedback}

If the state $x$ of the plant is not available for measurement, then consider a controller $K$ for the system (1) of the following form

$$
\begin{aligned}
\dot{\xi} & =a(\xi)+b(\xi) y \\
u & =c(\xi)
\end{aligned}
$$

such that the resulting closed-loop system has locally robust disturbance attenuation performance, where $\xi \in$ $R^{v}$.

Consider an auxiliary system $\Sigma_{0}$ given by

$$
\begin{aligned}
& \dot{x}=f(x)+g_{2}(x) u\left[\gamma \sqrt{\lambda_{1}(x)} f_{11}(x),\right. \\
& \left.+\gamma \sqrt{\lambda_{2}(x)} g_{11}(x), 0, g_{1}(x)\right] \hat{w} \\
& =f(x)+g_{10}(x) \hat{w}+g_{2}(x) u \\
& \hat{z}=\left[\begin{array}{c}
\frac{1}{\sqrt{\lambda_{1}(x)}} f_{22}(x) \\
0 \\
\frac{1}{\sqrt{\lambda_{3}(x)}} h_{22}(x) \\
h_{1}(x)
\end{array}\right]+\left[\begin{array}{c}
0 \\
\frac{1}{\sqrt{\lambda_{2}(x)}} g_{22}(x) \\
0 \\
k_{12}(x)
\end{array}\right] u \\
& =h_{10}+k_{120}(x) u \\
& y=h_{2}(x)+\left[\begin{array}{llll}
0 & 0 & \gamma \sqrt{\lambda_{3}(x)} h_{11}(x) & k_{21}(x)
\end{array}\right] \hat{w} \\
& =h_{2}(x)+k_{210}(x) \hat{w}
\end{aligned}
$$

Applying the controller $K$ to the system (1) and the system $\Sigma_{0}$, respectively, we obtain the following two closed-loop systems.

$$
\Sigma_{c 1}:\left\{\begin{array}{l}
\dot{x}_{e}=f_{e}\left(x_{e}\right)+\Delta f_{e}\left(x_{e}\right)+g_{e}\left(x_{e}\right) w \\
z=h_{1}(x)+k_{12}(x) c(\xi)
\end{array}\right.
$$

where $x_{e}=\left[\begin{array}{ll}x^{T} & \xi^{T}\end{array}\right]^{T}$,

$$
\begin{aligned}
& f_{e}\left(x_{e}\right)=\left[\begin{array}{c}
f(x)+g_{2}(x) c(\xi) \\
a(\xi)+b(\xi) h_{2}(x)
\end{array}\right] \\
& \triangle f_{e}\left(x_{e}\right)=\left[\begin{array}{c}
\triangle f(x)+\triangle g_{2}(x) c(\xi) \\
b(\xi) \triangle h_{2}(x)
\end{array}\right] \\
& g_{e}\left(x_{e}\right)=\left[\begin{array}{c}
g_{1}(x) \\
b(\xi) k_{21}(x)
\end{array}\right] \\
& \Sigma_{c 0}: \begin{cases}\dot{x}_{e} & =f_{e 0}\left(x_{e}\right)+g_{e 0}\left(x_{e}\right) \hat{w} \\
\hat{z} & =h_{10}(x)+k_{120}(x) c(\xi)\end{cases}
\end{aligned}
$$

where

$$
\begin{aligned}
& f_{e 0}\left(x_{e}\right)=f_{e}\left(x_{e}\right) \\
& g_{e 0}\left(x_{e}\right)=\left[\begin{array}{c}
g_{10}(x) \\
b(\xi) k_{210}(x)
\end{array}\right]
\end{aligned}
$$

Let $U\left(x_{e}\right)$ be a $C^{1}$ function defined in a neighbourhood of $(0,0)$, denote

$$
\begin{gathered}
J_{1}\left(U, \Sigma_{c 1}, \Delta f, \triangle g_{2}, \Delta h_{2}\right) \triangleq U_{x_{e}}\left(f_{e}\left(x_{e}\right)+\triangle f_{e}\left(x_{e}\right)\right) \\
+z^{T} z+\frac{1}{4 \gamma^{2}} U_{x_{e}} g_{e}\left(x_{e}\right) g_{e}^{T}\left(x_{e}\right) U_{x_{e}}^{T} \\
J_{0}\left(U, \Sigma_{c 0}, \lambda_{1}(x), \lambda_{2}(x), \lambda_{3}(x)\right) \triangleq U_{x_{e}}\left(f_{e 0}\left(x_{e}\right)+\hat{z}^{T} \hat{z}\right. \\
+\frac{1}{4 \gamma^{2}} U_{x_{e}} g_{e 0}\left(x_{e}\right) g_{e 0}^{T}\left(x_{e}\right) U_{x_{e}}^{T}
\end{gathered}
$$

Then the-functions $J_{1}$ and $J_{0}$ have the following relation. 
Lemma 3.1 For any $\triangle f(x) \in \Omega_{f}, \Delta g_{2}(x) \quad \epsilon$ $\Omega_{g}, \triangle h_{2}(x) \in \Omega_{h}$, and $\lambda_{1}(x)>0, \lambda_{2}(x), \lambda_{3}(x)>0$, the following inequality holds

$J_{1}\left(U, \Sigma_{c 1}, \triangle f, \triangle g_{2}, \Delta h_{2}\right) \leq J_{0}\left(U, \Sigma_{c 0}, \lambda_{1}(x), \lambda_{2}(x), \lambda_{3}(x)\right)$

Proof: Omitted due to space limitation.

$\triangleleft$

Remark 3.3 From the inequality (32) and the results in [11], we know that if there exist positive smooth functions $\lambda_{1}(x), \lambda_{2}(x)$ and $\lambda_{3}(x)$ such that the closedloop system $\Sigma_{c 0}$ of (27) is dissipative, with a $C^{T}$ storage function $U\left(x_{e}\right)$, with respect to the supply rate $s(\hat{w}, \hat{z})=\gamma^{2}\|\hat{w}\|^{2}-\|\hat{z}\|^{2}$, then the closed-loop system $\Sigma_{c 1}$ of (23) also is dissipative, with the same storage function $U\left(x_{e}\right)$, with respect to the supply rate $s(w, z)=\gamma^{2}\|w\|^{2}-\|z\|^{2}$ for any $\Delta f(x) \in \Omega_{f}$, $\triangle g_{2}(x) \in \Omega_{g}$ and $\triangle h_{2}(x) \in \Omega_{h}$.

Combining the above lemma, and Theorem 3.1 on [4], we have the following theorem.

Theorem 3.1 Consider the system (1) and the system $\Sigma_{0}$ of (22), $\lambda_{1}(x), \lambda_{2}(x)$ and $\lambda_{3}(x)$ are given positive scalar smooth functions, and suppose the following:

(i): Assumptions A2 and A3 hold, and the matrix $k_{12}^{T}(x) k_{12}(x)$ is nonsingular for each $x$,

(ii): There exists a $C^{3}$ positive definite function $V(x)$, locally defined in a neighbourhood of $x=0$ and vanishing at $x=0$, which satisfies the following inequality

$$
\begin{gathered}
H_{0}\left(x, V_{x}, \lambda_{1}(x), \lambda_{2}(x), \lambda_{3}(x)\right)=V_{x} \hat{f}(x) \\
+\hat{h}_{1}^{T}(x) \hat{h}_{1}(x)+\frac{1}{4} V_{x} \hat{R}(x) V_{x}^{T} \leq 0
\end{gathered}
$$

where

$$
\begin{gathered}
\hat{f}(x)=f(x)-g_{2}(x) R_{2}^{-1}(x) k_{120}^{T}(x) h_{10}(x) \\
\hat{h}_{1}(x)=h_{10}(x)-k_{120}(x) R_{2}^{-1}(x) k_{120}^{T}(x) h_{10}(x) \\
\hat{R}(x)=\frac{1}{\gamma^{2}} g_{10}(x) g_{10}^{T}(x)-g_{2}(x) R_{2}^{-1}(x) g_{2}^{T}(x)
\end{gathered}
$$

Denote

$$
\begin{gathered}
\alpha(x)=-R_{2}^{-1}(x)\left(\frac{1}{2} g_{2}^{T}(x) V_{x}^{T}+k_{120}^{T}(x) h_{10}(x)\right) \\
=-R_{2}^{-1}(x)\left(\frac{1}{2} g_{2}^{T}(x) V_{x}^{T}+k_{12}^{T}(x) h_{1}(x)\right) \\
\alpha_{1}(x)=\frac{1}{2 \gamma^{2}} g_{10}^{T}(x) V_{x}^{T} \\
f_{*}(x)=f(x)+g_{10} \alpha_{1}(x) \\
h_{2 *}(x)=h_{2}(x)+k_{210}(x) \alpha_{1}(x)
\end{gathered}
$$

(iii): There exists a $C^{3}$ positive definite function $Q(x)$, locally defined in a neighbourhood of $x=0$ and vanishing at $x=0$, which satisfies the following inequality

$$
\begin{gathered}
F_{0}\left(x, Q_{x}, \lambda_{1}(x), \lambda_{2}(x), \lambda_{2}(x)\right)=Q_{x} \hat{f}_{0}+\hat{T}(x) \\
+\frac{1}{4 \gamma^{2}} Q_{x} \hat{g}_{1}(x) \hat{g}_{1}^{T}(x) Q_{x}^{T}<0 \text { for } x \neq 0
\end{gathered}
$$

and the Hessian matrices of $F_{0}\left(x, Q_{x}, \lambda_{1}(x), \lambda_{2}(x)\right.$ and $\left.\lambda_{2}(x)\right)$ are nonsingular, where

$$
\begin{gathered}
\hat{f}_{0}(x)=f_{*}(x)-g_{10}(x) k_{210}^{T}(x) R_{1}^{-1}(x) h_{2 *}(x) \\
\hat{g}_{1}(x)=g_{10}(x)\left[I-k_{210}^{T}(x) R_{1}^{-1}(x) k_{210}(x)\right] \\
\hat{T}(x)=\alpha^{T}(x) R_{2}(x) \alpha(x)-\gamma^{2} h_{2 *}^{T}(x) R_{1}^{-1}(x) h_{2 *}(x)
\end{gathered}
$$

with

$$
R_{1}(x)=\gamma^{2} \lambda_{3}(x) h_{11}(x) h_{11}^{T}(x)+k_{21}(x) k_{21}^{T}(x)
$$

(iv): There exists a matrix function $L(x)$ such that

$$
Q_{x} L(x)=h_{2 *}^{T}(x)
$$

Denote

$$
\begin{aligned}
G(x) & =\left(2 \gamma^{2} L(x)+g_{10}(x) k_{210}^{T}(x)\right) R_{1}^{-1}(x) \\
& =\left(2 \gamma^{2} L(x)+g_{1}(x) k_{21}^{T}(x)\right) R_{1}^{-1}(x)
\end{aligned}
$$

Then, the controller $K$ given by (21) with

$$
\begin{aligned}
& a(\xi)=f_{*}(\xi)+g_{2}(\xi) \alpha(\xi)-G(\xi) h_{2 *}(\xi) \\
& b(\xi)=G(\xi), \quad c(\xi)=\alpha(\xi)
\end{aligned}
$$

is a solution of the robust disturbance attenuation problem via measurement feedback for the system (1).

Theorem 3.2 Consider the system (1) and suppose the following:

(i'): Assumptions A1 and A3 hold,

(ii') There exists a $C^{3}$ positive definite function $V(x)$, locally defined in a neighbourhood of $x=0$ and vanishing at $x=0$, which satisfies the following strict inequality

$$
H_{0}\left(x, V_{x}, \lambda_{1}(x), \lambda_{2}(x), \lambda_{3}(x)\right)<0 \text { for } x \neq 0
$$

(iii'): (iii) and (iv) in Theorem 3.1 hold.

Then, the controller $K$ given by (21) with (48) and (49) is a solution of the robust disturbance attenuation problem via measurement feedback for the system (1).

Proofs for Theorems 3.1 and 3.2 are omitted here due to space limitations. 
Remark 3.4 From the above results, it is easy to see that the solution of the robust disturbance attenuation problem via measurement feedback for the uncertain system (1) can be conducted by the extended system $\Sigma_{0}$ without uncertainty, which is similar to the result for linear systems in [14]. For the case of state feedback, the similar conclusion holds for the system (1) and the extended system $\Sigma_{s}$ of (16), i.e., the controller given in Theorem 2.1 or Theorem 2.2 can be conducted by the extended system $\Sigma_{s}$, but the proofs of Theorem 2.1 and Theorem 2.2 are direct from the standard "completion of the squares" argument.

Remark 3.5 By computing directly, we have

$$
\begin{gathered}
H_{s}\left(x, V_{x}^{T}, \lambda_{1}(x), \lambda_{2}(x)\right)=H_{0}\left(x, V_{x}^{T}, \lambda_{1}(x), \lambda_{2}(x), \lambda_{3}(x)\right) \\
-\frac{1}{\lambda_{3}(x)} h_{22}^{T}(x) h_{22}(x)
\end{gathered}
$$

which is different from the $H_{\infty}$ control system design for affine nonlinear systems without uncertainty. This is caused by the uncertainty of the measured output.

Remark 3.6 For the linear case, the similar can be made as Corollary 2.1 and Remark 2.2, the details are omitted.

\section{Conclusions}

This paper addresses the robust $H_{\infty}$ control problems via state feedback and measurement feedback for a class of uncertain affine nonlinear systems. The proposed solutions rely upon the existence of positive definite smooth solutions of Hamilton-Jaccobi inequalities, which can be conducted by solving the corresponding $H_{\infty}$ control problem for the extended systems without uncertainty.

\section{References}

[1] J.Ball, J.W. Helton, and M.L. Walker, " $H_{\infty}$ control for nonlinear systems via output feedback", IEEE Trans. Automat. Contr., Vol. 38, 546-559, 1993

[2] J.C. Doyle, K. Glover, P.P. Khargonekar and B.A. Francis, "State space solutions to standard $H_{2}$ and $H_{\infty}$ control problems", IEEE Trans. Automat. Contr., Vol. 34, 831-847, 1989.

[3] A. Isidori and A. Astolfi, "Disturbance attenuation and $H_{\infty}$ control via measurement feedback in nonlinear systems", IEEE Trans. Automat. Contr., Vol. 37, 1283-1293, 1992.

[4] A. Isidori, " $H_{\infty}$ control via measurement feedback for affine nonlinear systems", Int. J. Robust and Nonlinear Control, Vol. 4, 553-574, 1994.
[5] A. Isidori, "A necessary condition for nonlinear $H_{\infty}$ control via measurement feedback", Systems Control Lett., Vol. 23, 169-177, 1994.

[6] R. Marino and P. Tomei, "Robust stabilization of feedback linearizable time-varying uncertain nonlinear systems", Automatica, Vol. 29, 181-189, 1993.

[7] H. Nijmeijer and A.J. Van der Schaft, Nonlinear Dynamical Control Systems, Springer-Verlag: New York, 1990.

[8] I.R. Petersen, "A stabilization algorithm for a class of uncertain linear systems", Systems Control Lett., Vol. 8, 351-357, 1987.

[9] T. Shen and K. Tamura, "Robust $H_{\infty}$ control of uncertain nonlinear systems via state feedback", IEEE Trans. Automat. Contr., Vol. 40, 766-768, 1995.

[10] A.J. Van der Schaft, "A state-space approach to nonlinear $H_{\infty}$ control", Systems Control Lett., Vol. 16, $1-8,1991$.

[11] A.J. Van der Schaft, " $L^{2}$-gain analysis of nonlinear systems and nonlinear $H_{\infty}$ control", IEEE Trans. Automat. Contr., Vol. 37, 770-784, 1992.

[12] Y. Wang, L. Xie, and C.E. de Souza, "Robust control of a class of uncertain nonlinear systems", Systems Control Lett., Vol. 19, 139-149, 1992.

[13] L. Xie and C.E. de Souza, "Robust $H_{\infty}$ control for linear systems with norm-bounded time-varying uncertainty", IEEE Trans. Automat. Contr., Vol, 37, 1188-1191, 1992.

[14] L. Xie, M. Fu, and C.E. de Souza, " $H_{\infty}$ control and quadratic stabilization of systems with parameter uncertainty via output feedback", IEEE Trans. Automat. Contr., Vol. 37, 1253-1256, 1992. 